\title{
DIEUDONNÉ-SCHWARTZ THEOREM ON BOUNDED SETS IN INDUCTIVE LIMITS
}

\section{J. KUCERA AND K. MCKENNON}

\begin{abstract}
The Dieudonné-Schwartz Theorem for bounded sets in strict inductive limits does not hold for general inductive limits $E=$ ind $\lim E_{n}$. It does if every closed convex set in $E_{n}$ is closed in $E_{n+1}$. This condition is not necessary. In case all spaces $E_{n}$ are normed a necessary and sufficient condition for the validity of the Dieudonne-Schwartz Theorem is given.
\end{abstract}

Let $E_{1} \subset E_{2} \subset \cdots$ be a sequence of locally convex spaces and $E=$ ind $\lim E_{n}$ their inductive limit (with respect to the identity maps id: $E_{n} \rightarrow E_{n+1}$ ). The Dieudonné-Schwartz Theorem (further referred to as DST), see [2, Chapter 2, §12], states that a set $B \subset E$ is bounded if and only if it is contained and bounded in some $E_{n}$, provided that

(H-1) each $E_{n}$ is closed in $E_{n+1}$ and

(H-2) the topology of each $E_{n}$ equals the topology induced in $E_{n}$ by $E_{n+1}$.

These two hypotheses imply [2, Chapter 2, §12]

(H-3) each $E_{n}$ is closed in $E$.

It is shown in [3] that if H-3 holds and $B$ is a bounded set in $E$, then $B \subset E_{n}$ for some $n$, but may not be bounded there. Therefore, in order to preserve the DST, we need a stronger hypothesis than $\mathrm{H}-3$. We introduce three more.

(H-4) each convex and closed set in $E_{n}$ is closed in $E_{n+1}$,

(H-5) for each set $B$ bounded and convex in $E_{n}$, the closure $\bar{B}^{E}$ of $B$ in $E$ is contained and bounded in $E_{n+p}$ for some $p \in N$,

(H-6) for each set $B$ bounded and convex in $E_{n}$, the closure $\bar{B}^{E}$ of $B$ in $E$ is contained in $E_{n+p}$ for some $p \in N$.

LEMMA 1 . $\mathrm{H}-4 \Rightarrow \mathrm{H}-3$.

Proof. Assume that $E_{1}$ is not closed in $E$ and $x \in \bar{E}_{1}^{E} \backslash E_{1}$. Since $E_{1}$ is closed in $E_{2}$, there exists a closed convex neighborhood $U_{2}$ of 0 in $E_{2}$ such that $x \notin E_{1}+$ $2 U_{2}$. Now, $\bar{E}_{1}+U_{2}^{E}$ is closed convex in $E_{2}$ and, by $\mathrm{H}-4$, closed in $E_{3}$. Since $\bar{E}_{1}+U_{2} E \subset E_{1}+2 U_{2}$, there exists a closed convex neighborhood $U_{3}$ of 0 in $E_{3}$ such that $x \notin E_{1}+U_{2}+2 U_{3}$. When all $U_{2}, U_{3}, \ldots$ are constructed, the set $E_{1}+\cup_{k=2}^{\infty}\left(U_{2}+U_{3}+\cdots+U_{k}\right)$ is a neighborhood of $E_{1}$ in $E$ which does not contain $x$, a contradiction.

THEOREM $1 . \mathrm{H}-4 \Rightarrow D S T$.

Received by the editors April 13, 1979.

AMS (MOS) subject classifications (1970). Primary 46A05.

Key words and phrases. Locally convex space, inductive limit, bounded set.

(c) 1980 American Mathematical Society $0002-9939 / 80 / 0000-0117 / \$ 01.75$ 
Proof. Let $B$ be a bounded set in $E$. According to Lemma 1 and [3], $B \subset E_{n}$ for some $n$. Put $n=1$ and assume $B$ is not bounded in any $E_{m}, m \in N$.

Since $E_{1}$ is a locally convex space, $B$ is not weakly bounded there and there exists a continuous linear functional $f_{1}: E_{1} \rightarrow R$ which is unbounded on $B$. Choose a sequence $\left\{b_{k}\right\} \subset B$ such that $f_{1}\left(b_{k}\right)>k, k=1,2, \ldots$ The set $U_{1}=\left\{x \in E_{1}\right.$; $\left.f_{1}(x) \leqslant 1\right\}$ is closed convex in $E_{1}$, hence closed in $E_{2}$, and there exists a continuous linear functional $g: E_{2} \rightarrow R$ such that $U_{1} \subset\left\{x \in E_{2} ; g(x)<1\right\}$ and $g\left(b_{1}\right)>1$.

If $f_{1}(x)=0$, then $f_{1}(k x)=0$ for every integer $k$ and $k x \in U_{1}$. This implies $g(k x)=0$ and $g(x)=0$. Hence $\left.g\right|_{E_{1}}=c f_{1}$, where $\left.g\right|_{E_{1}}$ is the restriction of $g$ to $E_{1}$. Then $f_{2}=g / c$ is a continuous extension of $f_{1}$ to $E_{2}$. The set $U_{2}=\left\{x \in E_{2}\right.$; $\left.f_{2}(x)<1\right\}$ is a closed convex neighborhood of 0 in $E_{2}$ for which $U_{1} \subset U_{2}$ and $b_{1} \notin U_{2}, b_{2} / 2 \notin U_{2}$.

Since $U_{2}$ is closed in $E_{3}$, the process can be repeated until we get a sequence $\left\{f_{k}\right.$ : $\left.E_{k} \rightarrow R ; k=1,2,3, \ldots\right\}$ of continuous linear functionals, each of which is an extension of its predecessor, and $b_{r} / r \notin U_{k}=f_{k}^{-1}(-\infty, 1]$ for $r=1,2, \ldots, k$. The set $U=\cup_{k=1}^{\infty} U_{k}$ is a neighborhood of 0 in $E$ and $B \subset s U$ for some $s \in N$. But $b_{s} / s \notin U$, which is a contradiction.

THEOREM 2. If all $E_{n}$ are normed spaces, then H-5 is equivalent to DST.

Proof. 1. Let DST hold and $B$ be bounded and convex in $E_{n}$. Then $B$ and $\bar{B}^{E}$ are bounded in $E$ and $\bar{B}^{E}$ must be bounded in some $E_{n+p}$. We did not need normability of the $E_{n}$ 's.

2. Let H-5 hold and $B$ be bounded in $E$ but not bounded in any $E_{n}$. Denote by $B_{n}$ the closed unit ball in $E_{n}$. There exists $b_{1} \in B \backslash\{0\}$ and a closed convex neighborhood $V_{1}$ of 0 in $E$ such that $b_{1} \notin V_{1}$. For some $p_{1} \in N, b_{1} \in E_{p_{1}}$. Put $U_{1}=\overline{V_{1} \cap B_{p_{1}}} E$. Then $U_{1} \subset V_{1}$ and $b_{1} \notin U_{1}$. Since $V_{1} \cap B_{p_{1}}$ is bounded and convex in $E_{p_{1}}, U_{1}$ is contained and bounded in some $E_{p_{2}}$. Hence there exists $b_{2} \in$ $B \backslash 2 U_{1}$. We may take $p_{2}$ so that $p_{2}>p_{1}$ and $b_{2} \in E_{p_{2}}$. Further, $U_{1}$ is closed and convex in $E$. Hence there exists a closed convex neighborhood $V_{2}$ of 0 in $E$ such that $b_{1}, \quad b_{2} / 2 \notin U_{1}+2 V_{2}$. Put $U_{2}=\overline{V_{2} \cap B_{p_{2}}}{ }^{2}$. Again, $U_{2} \subset V_{2}$ and $b_{1}$, $b_{2} / 2 \notin \overline{U_{1}+U_{2}}{ }^{E} \subset \overline{U_{1}+V_{2}}{ }^{E} \subset U_{1}+V_{2}+V_{2}$.

We repeat this process until we get sequences $\left\{b_{k}\right\} \subset B, p_{1}<p_{2}<\cdots$, and a sequence of closed convex neighborhoods $V_{1}, V_{2}, \ldots$ of 0 in $E$, such that $b_{k} / k \notin$ $U_{1}+U_{2}+\cdots+U_{n}$ for $k=1,2, \ldots, n$, where $U_{k}={\overline{V_{k} \cap B_{p_{k}}}}^{E}$. Then $U=$ $\bigcup_{k=1}^{\infty}\left(U_{1}+U_{2}+\cdots+U_{k}\right)$ is a neighborhood of 0 in $E$ and $B \subset s U$ for some $s$. But $b_{s} / s \notin U$, a contradiction.

With a slight modification of the last proof we can get

THEOREM 3. If all $E_{n}$ are normal spaces then H-6 is equivalent to: Each bounded set in $E$ is contained in some $E_{n}$.

Lemma 2. Let $X, Y$ be Banach spaces, $X \subset Y$, id: $X \rightarrow Y$ continuous, and $X$ reflexive. Then every bounded closed convex set in $X$ is closed in $Y$.

The proof follows from the Alaoglu Theorem.

THEOREM 4. If all $E_{n}$ are reflexive Banach spaces, then DST holds. 
Proof. It is sufficient to show that $\mathrm{H}-5$ holds. Let $B$ be a bounded closed convex set in $E_{n}$ and $b \notin B$. There exists a bounded closed convex neighborhood $U_{0}$ of 0 in $E_{n}$ such that $b \notin B+U_{0}$. By Lemma 2, $B+U_{0}$ is bounded closed and convex in $E_{n+1}$. Hence there is a bounded closed convex neighborhood $U_{1}$ of 0 in $E_{n+1}$ such that $b \notin B+U_{0}+U_{1}$, etc. The set $U=\cup_{k=0}^{\infty}\left(U_{0}+U_{1}+\cdots+U_{k}\right)$ is a neighborhood of 0 in $E$ and $b \notin B+U$, i.e. $B$ is closed in $E$.

EXAMPLE. Let $R_{+}=[0, \infty), w_{n}(x)=\exp x / n, x \geqslant 0, E_{n}=\left\{f \in L^{2}\left(R_{+}\right)\right.$; $\left.\left\|w_{n} f\right\|_{2}<+\infty\right\}, n \in N$. All $E_{n}$ are Hilbert spaces with the inner product $(f, g) \mapsto$ $\left\langle w_{n} f, w_{n} g\right\rangle_{2}, E_{1} \subset E_{2} \subset \cdots$, and id: $E_{n} \rightarrow E_{n+1}$ are continuous. By Theorem 4, DST holds. We show that $\mathrm{H}-1$, and hence both $\mathrm{H}-3$ and $\mathrm{H}-4$, do not hold. It means that $\mathrm{H}-4$ is not a necessary condition in Theorem 1.

Take $n \in N$ and $1 /(n+1)<a<b<1 / n$. Then $\exp (-a x) \in E_{n+1} \backslash E$. The functions

$$
f_{k}(x)= \begin{cases}\exp (-a x) & \text { for } 0 \leqslant x \leqslant k \\ \exp (-b x) & \text { for } k \leqslant x\end{cases}
$$

all belong to $E_{n}$ and converge in $E_{n+1}$ to $\exp (-a x)$.

\section{REFERENCES}

1. J. Dieudonné and L. Schwartz, La dualité dans les espaces (F) et (LFF), Ann. Inst. Fourier (Grenoble) 1 (1949), 61-101.

2. J. Horváth, Topological vector spaces and distributions, Vol. 1, Addison-Wesley, Reading, Mass., 1966.

3. J. Kucera and K. McKennon, Bounded sets in inductive limits, Proc. Amer. Math. Soc. 69 (1978), $62-64$.

Department of Mathematics, Washington State University, Pullman, Washington 99164 